\title{
Medienwissen und Medienmenüs als kulturelles Kapital und als Distinktionsmerkmale
}

\author{
Eine Typologie der Mediennutzer in Deutschland
}

\section{Michael Meyen}

Warum verbringen die Deutschen inzwischen im Durchschnitt jeden Tag zebn Stunden mit den Angeboten der Massenmedien? Warum werden einige Menschen zu Informationsjunkies und andere zu Medienvermeidern? Von welchen Faktoren hängt das Medienmenü ab, das ein einzelner Nutzer konsumiert? Die Studie, die sich auf 133 Tiefeninterviews stützt, gebt in Anlehnung an Pierre Bourdieu davon aus, dass der Habitus und damit die Mediengewohnheiten vor allem von der sozialen Position eines Akteurs bestimmt werden, und fragt danach, wie die zehn Stunden, die der deutsche Durchschnittsbürger jeden Tag mit den Angeboten der Massenmedien verbringt, zum individuellen Kapitalfundus beitragen. Die Untersuchung zeigt, dass Medienwissen und Medienmenüs zu Distinktionsmerkmalen geworden sind und kulturelles Kapital liefern. Mediennutzung ist für viele Menschen folglich (auch) Arbeit. Der Norm, informiert zu sein, können sich vor allem diejenigen nicht entzieben, die eine gehobene soziale Position bekleiden oder aufsteigen wollen. Mindestens genauso wichtig wie die soziale Position ist dabei die Bewertung, die der jeweilige Akteur mit dieser Position verbindet. Dieses Urteil wiederum wird vor allem vom Geschlecht, von der Herkunft, der Lebensphase, dem persönlichen Umfeld und der Alltagsbelastung beeinflusst.

Schlagwörter: Mediennutzung, Medienmenüs, Bourdieu, Qualitative Forschung, Leitfadeninterviews, Typologie

Die akademische Medienforschung behauptet zwar, sie habe die Frage „Was machen die Medien mit den Menschen?“ schon vor mehr als drei Jahrzehnten umgedreht (vgl. Blumler \& Katz, 1974; Jensen \& Rosengren, 1990), in der Forschungspraxis aber dominiert eine psychologische Sichtweise, die sich auf einzelne Medienangebote, auf spezielle Rezeptionssituationen und auf die analytische Mikroebene konzentriert (vgl. exemplarisch die Beiträge in Wirth, Schramm \& Gehrau, 2006; Bryant \& Vorderer, 2006 und Rössler, Kubisch \& Gehrau, 2002). Dies gilt auch dort, wo die Forschung „transmedial“ ausgerichtet ist (Schweiger, 2005) oder wo sie den Alltag thematisiert, in den Mediennutzung eingebettet ist, sowie den Stellenwert, den die jeweiligen Angebote in diesem Alltag haben (vgl. Huber \& Meyen, 2006; Weiß, 2001; Hepp, 1998; Cornelißen, 1998; Mikos, 1994; Charlton \& Neumann-Braun, 1988; Bausinger, 1984). Auf diese Weise sind zwar sehr differenzierte Motivkataloge und ein enormes Detailwissen über Rezeptionsvorgänge und über die Aneignung von Medientexten entstanden, die Frage aber, warum die Deutschen sich inzwischen im Durchschnitt jeden Tag zehn Stunden lang mit den Angeboten der Massenmedien beschäftigen und damit fast doppelt so lange wie vor einem Vierteljahrhundert (Reitze \& Ridder, 2006: 49f.), kann so ebenso wenig beantwortet werden wie die Frage nach individuellen Unterschieden: Warum werden einige Menschen zu Informationsjunkies, andere zu Spezialisten im Unterhaltungsslalom und manche zu Medienvermeidern?

Die vorliegende Studie zielt auf diese Forschungslücken und damit auf die Makroebene. Wenn hier die Kapital-Theorie Bourdieus als Heuristik genutzt wird, geht es folglich nicht darum, neue Nutzungsmotive zu konstruieren (etwa: Akkumulation 
von kulturellem Kapital). Vorgeschlagen wird vielmehr eine Perspektive, die die vielen Einzelbefunde integrieren und zugleich erklären kann, warum die Nutzung von Medienangeboten den Alltag in modernen Gesellschaften dominiert und welche Faktoren die Zusammenstellung eines Medienmenüs beeinflussen (das, was ein einzelner Nutzer konsumiert). Die Studie geht in Anlehnung an den Uses-and-Gratifications-Approach davon aus, dass Mediennutzung sowohl von den Bedürfnissen abhängt, die sich aus der jeweiligen sozialen und psychologischen Situation ergeben, als auch von den Erfordernissen und den Strukturen des Alltags (Rubin, 2002). Da diese Situation, die mit der ganz persönlichen Lebensgeschichte zusammenhängt, und der Alltag kaum standardisiert zu erfassen sind und da die Erkundung von Motiven schwierig ist, wurden Leitfadeninterviews durchgeführt. Die 133 Befragten sind nach dem Verfahren der theoretischen Sättigung ausgewählt worden (Grundgesamtheit: erwachsene Deutsche, die nicht mehr zur Schule gehen).

Dass der Beitrag nicht „klassisch“ aufgebaut ist (Frage, Theorie, Empirie, Interpretation), sondern mit einer Diskussion des Untersuchungsdesigns beginnt, lässt sich mit dem Forschungsprozess begründen. Theoretischer Ausgangspunkt war ursprünglich Rosengrens Lebensstil-Modell (Rosengren, 1994; 1995). Dieses Modell nimmt an, dass alle Handlungsmuster (und damit auch die Nutzung von Medienangeboten) von strukturellen, positionellen und individuellen Faktoren und Bedingungen beeinflusst werden. Das Untersuchungsmaterial selbst hat es dann nahegelegt, diese Perspektive auszubauen und die Interpretation der Transkripte auf die schon erwähnte Kapital-Theorie von Bourdieu zu stützen. Da diese Entscheidung erst während der Auswertung gefallen ist und so an das Verfahren erinnert, das unter dem Begriff Grounded Theory bekannt geworden ist (Krotz, 2005), wird die eigentliche theoretische Grundlegung erst im zweiten Abschnitt geleistet - als Teil der Ergebnisaufbereitung.

\section{Untersuchungsdesign}

Die wichtigsten theoretischen Vorentscheidungen (Orientierung am Uses-and-Gratifications-Approach sowie an Rosengrens Lebensstil-Modell) und die Methode (Leitfadeninterviews) sind bereits in der Einleitung mit Blick auf den Gegenstand der Untersuchung knapp begründet worden. Qualitative Methoden sind standardisierten Verfahren bei der Frage, welche Bedeutung Medienangebote für die Menschen haben, überlegen, weil die Befragten hier die Möglichkeit haben, frei über ihren Lebenslauf und ihren Alltag, über ihr persönliches Umfeld und ihre Bedürfnisse zu sprechen, und so Hinweise für die Interpretation ihrer Mediengewohnheiten liefern. Tiefeninterviews können allerdings nicht repräsentativ sein und niemals Aufschluss über die Verteilung von bestimmten Handlungsmustern in der Grundgesamtheit geben, sondern bestenfalls typische Varianten ohne Anspruch auf Vollständigkeit beschreiben (Fuchs-Heinritz, 2000). Diese Methode setzt die Bereitschaft und die Fähigkeit voraus, einem Fremden etwas aus seinem Leben zu erzählen.

\subsection{Auswabl der Befragten}

Um trotzdem nicht auf Verallgemeinerungen verzichten zu müssen, sind die Befragten nach dem Verfahren der „theoretischen Sättigung“ ausgewählt worden. Dieses Verfahren geht davon aus, dass es bei einem Handlungsbereich wie Mediennutzung nicht unendlich viele Spielarten gibt. Um den Bereich beschreiben zu können, müssen die Befragten für möglichst unterschiedliche Varianten stehen, wobei die Annahmen, die die 
Auswahl bestimmen, so lange ergänzt und angepasst werden, bis die „neuen Fälle“ keine zusätzlichen Informationen mehr liefern. Die Auswahlkriterien wurden dabei zunächst Rosengrens Lebensstil-Modell entnommen (vgl. Abbildung 1). Rosengren hat dieses Modell vor allem entwickelt, um den Einfluss individueller Merkmale messen und die Frage beantworten zu können, welchen Spielraum der Mensch bei der Gestaltung seines Lebens hat. Als Lebensstile definierte er solche Handlungsmuster, die nicht von der gesellschaftlichen Struktur oder von der Position bestimmt sind, die der Mensch gerade besetzt, sondern vom Einzelnen in Übereinstimmung mit seinen Werten und Überzeugungen selbst gestaltet werden (Rosengren, 1995: 14-16). Damit lässt sich dieser Ansatz zwar in den Trend der individuellen Mediennutzungsforschung einordnen, bietet aber zugleich Anknüpfungspunkte für die Makroperspektive, um die es in dieser Studie ging. Abbildung 1 verdeutlicht zum einen die überragende Bedeutung struktureller Merkmale, die nicht nur alle Handlungsmuster mitbestimmen, sondern auch in positionelle und individuelle Merkmale einfließen, und gibt zum anderen erste Hinweise auf Determinanten der Mediennutzung und damit auf Ursachen für den individuell unterschiedlichen Umgang mit Medienangeboten. Das Wort Hinweise wurde gewählt, weil Rosengren diesen Punkt in seinen Veröffentlichungen nicht weiter ausgeführt hat und weil in den empirischen Arbeiten des schwedischen Media Panel Program, in dem das Modell entwickelt und genutzt wurde, stets nur einige ausgewählte Variablen gemessen oder getestet worden sind (vgl. exemplarisch Johansson \& Miegel, 1992). Wenn in diesem Beitrag nach den Faktoren gefragt wird, die die Zusammenstellung eines Medienmenüs beeinflussen, dann steht dahinter auch ein theoretisches Interesse: Welche Merkmale und Bedingungen hängen mit dem Handlungsmuster Mediennutzung zusammen?

\section{Abbildung 1: Determinanten von Handlungsmustern (Rosengren 1994; 1995)}

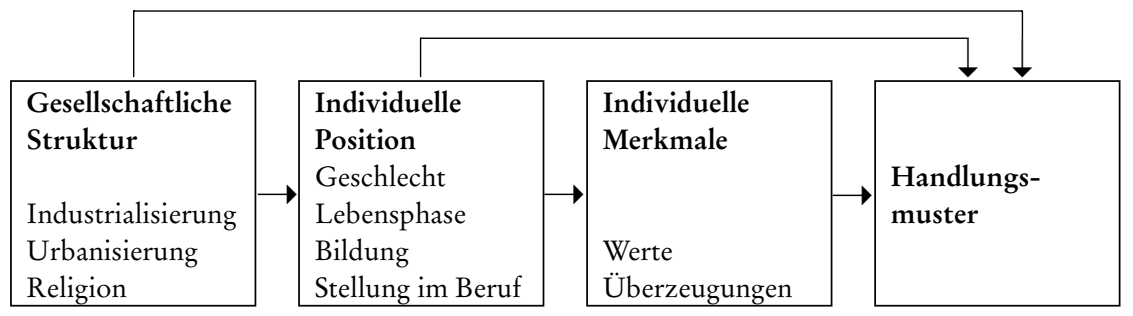

Da die wichtigsten strukturellen Bedingungen für die Menschen in Deutschland weitgehend ähnlich sein dürften (Ausnahmen: das Stadt-Land- sowie das West-Ostbzw. das Süd-Nord-Gefälle) und da individuelle Merkmale wie Werte, Überzeugungen oder die persönliche Lebensgeschichte vorab nicht ohne Aufwand zu ermitteln sind, hat das Auswahlverfahren bei zentralen positionellen Merkmalen der Befragten angesetzt: Geschlecht, Lebensphase, Einkommen, Stellung im Beruf. Auf diese Weise konnten auch strukturelle und individuelle Unterschiede eingefangen werden, weil der Einfallsreichtum der Studierenden, die an dem Projekt „Medien im Alltag“ beteiligt waren, bei der Suche nach „neuen Fällen“ so gut wie keine Grenzen kannte. Es wurden Rentner befragt und Mütter mit Kleinkindern, Lehrerinnen aus Schleswig-Holstein und Verkäuferinnen aus Baden-Württemberg, Polizisten, Arbeitslose und Politiker, Hochbegabte, Klassikhörer und Hörspielfans, Pfarrer, Ordensleute und Frauen in Führungspositionen, taz-Abonnenten, Leser von Wirtschaftszeitungen und Journalisten. Eine solche bewusste Auswahl ist, das sei wiederholt, niemals repräsentativ. In der Stichprobe sind 
zum Beispiel Berliner und Norddeutsche deutlich unterrepräsentiert. Das Prinzip der „theoretischen Sättigung“ hat es außerdem mit sich gebracht, dass längst nicht alle Tiefeninterviews in die Auswertung einfließen mussten. Schon nach wenigen Gesprächen mit jungen Müttern war zum Beispiel klar, dass kleine Kinder jeden Alltag dominieren und damit auch die Nutzung von Medienangeboten - weitgehend unabhängig vom Partnerschaftsmodell, von der Wohn- und Arbeitsform und auch davon, ob Großeltern in der Nähe sind oder nicht. Bei den befragten Journalisten war es genau umgekehrt: In diesem Beruf wird die Mediengesellschaft gelebt, und die wenigen Ausnahmen (zum Beispiel freiberufliche Reporterinnen, die kleine Kinder haben), waren schnell gefunden. Von den etwas mehr als 400 Interviews, die zwischen 2003 und Anfang 2006 geführt worden sind, wurden am Ende 133 für die Typologie verwendet, die in Abschnitt 3 präsentiert wird. Die wichtigsten soziodemographischen Angaben zu dieser Stichprobe:

- Geschlecht: 65 Frauen, 68 Männer;

- Alter: 15 Personen über 70 Jahre, 38 Personen zwischen 50 und 70, 53 Personen zwischen 30 und 50, 27 Personen jünger als 30 Jahre, aber älter als 20. Interviews mit Schülern und Lehrlingen haben gezeigt, dass der Zugang zu den Medien vom Elternhaus und vom Geldbudget reglementiert wird und sich deshalb in dieser Lebensphase nur bedingt eigenständige Medienmenüs herausbilden können;

- Bildung: 62 Personen mit Hochschulabschluss (bedingt vor allem durch die höhere Teilnahmebereitschaft);

- Beruf: 36 Arbeiter, Angestellte und Beamte, 16 leitende Angestellte, 15 Unternehmer, 17 Künstler und Freiberufler, 10 Studenten, 9 Hausfrauen, Arbeitslose und Langzeitpatienten, 30 Rentner.

\subsection{Interviewervorbereitung und Leitfaden}

Tiefeninterviews stellen hohe Anforderungen an die Gesprächsleiter. Sie müssen allgemein formulierte Forschungsfragen spontan auf die Situation und den Befragten anwenden. Der Erfolg der Interviews hängt außerdem stärker als bei standardisierten Verfahren davon ab, ob die ,Chemie' zwischen den Gesprächspartnern stimmt. Dies gilt besonders, wenn Mittel- und Oberschichtangehörige (zu denen Studierende in der Regel gehören) mit so genannten einfachen Leuten sprechen und junge Menschen mit sehr viel älteren. Gerade bei den Interviews mit Senioren war es oft nicht leicht, den Redefluss einzudämmen und auf das Thema Mediennutzung hinzulenken. Die Interviewer wurden auf solche Probleme in einem Hauptseminar vorbereitet, das jeweils über ein Semester lief. Bedingung bei der Rekrutierung war, dass sich die Gesprächspartner vorher nicht kannten. Die Probleme, die in der Methodenliteratur unter dem Stichwort „soziale Erwünschtheit" diskutiert werden und die beim Thema Mediennutzung vor allem mit dem Prestige der einzelnen Angebote zusammenhängen, treten vor allem dann auf, wenn sich Interviewer und Befragter wiedersehen werden oder wenn die Gefahr besteht, dass der Inhalt an gemeinsame Bekannte weitergegeben wird (vgl. Noelle-Neumann \& Petersen 1998: 62). Vollständig lösen ließ sich dieses Problem nicht. Damit die Zusage für das Interview eine gewisse Verbindlichkeit hat, wurde der Kontakt über Dritte hergestellt - über Menschen, denen sich der gesuchte Interviewpartner stark genug verpflichtet fühlte, um die Teilnahmebarrieren zu überwinden.

Um sicherzustellen, dass tatsächlich über Mediengewohnheiten gesprochen wird, sind die Interviews mit einem Leitfaden strukturiert worden. Dieser Leitfaden hat nur vorgegeben, welche Themen überhaupt berührt werden sollten, und den Befragten damit erlaubt, ihre eigene Sicht der Dinge darzulegen, Episoden anzubringen, Schwerpunkte 
zu setzen. Die vier Themenkomplexe des Leitfadens lassen sich sowohl mit den Basisannahmen und den Erkenntnissen der Uses-and-Gratifications-Forschung begründen als auch mit Rosengrens Vermutung, dass neben strukturellen und positionellen Faktoren auch individuelle Merkmale und Bedingungen den Umgang mit Medienangeboten beeinflussen:

- Lebenslauf und aktuelle Lebenssituation: Herkunft und Sozialisation, Alltagsstrukturen (Arbeit, Freizeit), Wohnung, Familie, Einkommen und Bildung, Bewertung des eigenen Lebens;

- Medienausstattung, Zugang zu Medienangeboten: Geräte, empfangbare Programme, Abonnements und regelmäßig gekaufte Zeitungen und Zeitschriften;

- Nutzungsmuster und Nutzungsmotive: Da Medien oft beiläufig und habituell genutzt werden und den Alltag begleiten, wurde ein ,normaler Medientag' rekonstruiert (im Idealfall ,gestern') - vom Aufstehen bis zum Einschlafen. Die Interviewer wussten um die Schwierigkeiten bei der Erkundung von Motiven und haben deshalb versucht, indirekt und möglichst offen zu fragen, Motive zu projizieren und die Befragten auch zu provozieren;

- Bewertung des Medienangebots: Glaubwürdigkeit, Image.

\subsection{Auswertung des Materials}

Die Interviews wurden mit einem Tonband aufgezeichnet und in normales Schriftdeutsch übertragen. $\mathrm{Zu}$ jedem Gespräch gibt es außerdem ein Protokoll, in dem Auffälligkeiten und Informationen über den oder die Befragten festgehalten wurden. Dort erfährt man zum Beispiel, dass ein Barkeeper, Jahrgang 1975, während des Interviews Milch für sein Kind aufwärmen musste, am Thema stark interessiert war und vorher eine Hasch-Zigarette geraucht hat. Solche Informationen helfen bei der Interpretation des Materials.

Für die Auswertung von Interviewtranskripten bietet die Methodenliteratur wenig Hilfe. Einigkeit besteht offenbar nur darin, dass sich die Strategie an den jeweiligen Zielen zu orientieren habe und folglich jedem Projekt auf den Leib zu schneidern sei (Mayring, 2002; Lamnek, 1998). An dieser Stelle bieten Projekte, die mit qualitativen Verfahren arbeiten, die größte Angriffsfläche, da die Verfahren für Außenstehende häufig nicht nachvollziehbar sind und folglich beliebig wirken müssen. Um die Möglichkeiten auszureizen, die das Interviewmaterial bietet, und es zugleich „auswertbar" zu machen, wurde eine Mischung aus geschlossenen und offenen Kategorien entwickelt: Geschlossene Kategorien:

- Soziodemographie: Geschlecht, Alter, Wohnortgröße, Familienform, formale Bildung, Stellung im Beruf, Zeitbudget, Einkommen;

- Netzwerke (Familie, Freunde, Bekannte), (nicht-mediale) Informationsquellen, persönliche Lebensgeschichte, Medienerfahrungen;

- Medienzugang, Freizeitalternativen.

Offene Kategorien:

- Muster der Mediennutzung: Medienmenü, Motive für die Nutzung einzelner Angebote;

- Bewertung: Informations- und Unterhaltungsqualität, Bindung an einzelne Sendungen oder Titel, Bedeutung von Medienangeboten im Leben des Befragten;

- Besonderheiten, Episoden, methodische Probleme. 
Die ersten beiden offenen Kategorien laufen auf ein Kurzporträt des Befragten hinaus, auf eine Interpretation im Lichte der theoretischen Ansätze, die Mediennutzung erklären wollen (vor allem Identitäts-, Erregungs- und Einstellungstheorien). Die (tendenziell) geschlossenen Kategorien sollten dann helfen, Unterschiede zwischen den einzelnen Personen zu erklären. Während Medienzugang und Freizeitalternativen in der Terminologie von Rosengren strukturelle Merkmale sind, stehen die soziodemographischen Kategorien für positionelle und Netzwerke, Informationsquellen, Lebensgeschichte sowie Medienerfahrungen für individuelle Merkmale, die in jedes Handlungsmuster und damit auch in den Umgang mit Medienangeboten einfließen. Um bei der Interpretation der Mediennutzungsmuster den Faktor Subjektivität einzuschränken, sind die Interviewer gebeten worden, ihre Gespräche mit Hilfe des gleichen Kategoriensystems auszuwerten (in einer Haus- oder einer Examensarbeit) - ein Verfahren, das in der Methodenliteratur auch „konsensuelles Codieren“ genannt wird (Schmidt, 2005: 453).

\section{Mediennutzung, Kapitalerwerb und der Sinn für die eigene Position}

Dass das Interviewmaterial dann nahegelegt hat, die Kapital-Theorie als AuswertungsHeuristik zu nutzen, mag nach diesem Vorspann verwundern. Rosengren hat sein Lebensstil-Modell scharf von Bourdieus theoretischen Vorstellungen abgegrenzt und den französischen Soziologen im Spannungsfeld zwischen Struktur und Handlung auf der anderen Seite verortet. Bei Bourdieu seien individuelle Lebensstile durch die gesellschaftlichen Strukturen und durch die Position des Einzelnen determiniert (Rosengren, 1995: 22f.). Rosengren begründete dies mit Ort und Zeit der Bourdieuschen Arbeiten: Auf dem Höhepunkt des Marxismus in den 1960er Jahren und in einer stark geschichteten Gesellschaft wie in Frankreich sei es vollkommen verständlich gewesen, die Frage nach dem Spielraum eines Menschen eher zu verneinen (Rosengren, 1995: 24).

Vergleicht man beide Ansätze genauer, scheint der Gegensatz längst nicht mehr so scharf zu sein (vor allem dann nicht, wenn man sich von Bourdieus normativem Wissenschaftsverständnis, vom Klassenbegriff und vom Ziel löst, Gesellschaft verändern zu wollen). Zum einen geht Bourdieu zwar davon aus, dass der Habitus (und damit ein wichtiges Prinzip des Handelns) durch die Gesellschaft und durch die Position in der Sozialstruktur geprägt ist und dass die jeweils verfügbaren Ressourcen Handlungsgrenzen markieren, innerhalb dieser Grenzen aber gesteht er den Menschen sehr wohl Freiheit, Individualität und die Chance auf Innovationen zu (anders hätte er zum Beispiel seinen eigenen sozialen Aufstieg auch gar nicht erklären können; vgl. Bourdieu, 2002). Zum anderen lässt auch Rosengrens Modell keineswegs annehmen, dass wir vollkommen „frei“ entscheiden können. Auch hier sind alle Handlungen durch die Gesellschaft und durch die jeweilige Position zumindest vorbestimmt. Dass Rosengren die Befunde aus dem Media Panel Program in Schweden sehr vorsichtig interpretiert hat („There seems indeed to be some space for individually determined lifestyles“; Rosengren, 1995: 25), deutet in die gleiche Richtung: Es gibt Spielraum für Individualität (der unterschiedlich ausfällt und folglich untersucht werden kann), aber keine Wahlfreiheit unabhängig vom Arbeitsalltag, von Geld, Zeit und Wohnverhältnissen, unabhängig von der Herkunft, die den Bildungsweg vorzeichnet und den Zugang zu Berufen und Positionen mitbestimmt und deren Bedeutung deshalb auch im Alter nicht nachlässt, wie Schulze (2000) meint, der geschrieben hat, dass der Mensch im Lauf der Zeit immer mehr „Herr seiner Situation “ werde und relativ frei im „Raum der Möglichkeiten“ seinen eigenen Lebensentwurf wählen könne.

Bourdieus zentrale Begriffe Kapital und Habitus haben den Vorteil, anders als Ro- 
sengrens Modell nicht statisch und zeitlos zu sein (Rosengren, 1995: 15). Habitus lässt sich vielleicht am besten mit dem Wortpaar „sozialisierte Subjektivität“ übersetzen. Bourdieu beschreibt mit diesem Begriff die Dispositionen der Menschen und damit all das, was sie seit ihrer frühesten Kindheit geformt hat: Familie, Schule und Lebensbedingungen, Laufbahn, individuelle und kollektive Erfahrungen. Der Habitus legt fest, was möglich ist - wie ein Akteur die Welt wahrnimmt, wie er andere bewertet, welchen Geschmack er hat, wie er denkt und handelt und wie er seinen Körper präsentiert und sich bewegt (Bourdieu, 1976: 165-167; 1998a: 21, 41f.). Obwohl Bourdieu kein Wort über Mediennutzung geschrieben hat (auch nicht in seinem Essay, Über das Fernsehen'; vgl. Bourdieu, 1998b; Averbeck, 2003; Bastin, 2003), ist das Habitus-Konzept damit ein Schlüssel zu den Mediengewohnheiten und zu den Themen, für die sich ein Mensch interessiert. Dieses Konzept zielt zwar auf dauerhafte Wahrnehmungs-, Denk- und Handlungsschemata und betont, dass frühe Erfahrungen spätere formen, der Habitus ist aber nichts Starres und Unveränderliches, sondern wird immer wieder modifiziert. Entscheidende Determinante ist dabei die soziale Position des jeweiligen Akteurs. Bei Bourdieu ist der Kampf um Status geradezu ein Synonym für menschliches Leben überhaupt. Den Titel seines Hauptwerks ,Die feinen Unterschiede` (Bourdieu, 1987) hat er selbst so interpretiert, dass Existieren nichts anderes heiße als „sich unterscheiden, unterschiedlich sein“, wobei ein Unterschied erst dann sozial relevant sei, wenn er von jemandem wahrgenommen werde, der einen Unterschied machen könne, weil er über die entsprechenden Wahrnehmungskategorien verfüge (Bourdieu 1998a: 22). Bourdieu geht davon aus, dass wir vor allem deshalb handeln, um uns von anderen abzuheben - ein Prozess, der ständig läuft und der uns überhaupt nicht bewusst sein muss. Ob in der Familie oder im Beruf, in der Nachbarschaft oder im Sportverein, am Urlaubsort oder in der S-Bahn: Immer geht es darum, unsere Handlungs- und Profitchancen dadurch zu vergrößern, dass wir in der jeweiligen Hierarchie einen Platz möglichst weit oben einnehmen. Den Spielraum des Einzelnen beschreibt Bourdieu mit dem Begriff Kapital. Er unterscheidet vier Kapitalformen (Bourdieu, 1983):

- ökonomisches Kapital: materieller Reichtum - alles, was in Geld umtauschbar ist, und damit eigentlich auch Bücher oder Kunstwerke, die bei Bourdieu als „kulturelles Kapital“ in "objektiviertem Zustand" auftauchen;

- kulturelles Kapital: Fähigkeiten, Fertigkeiten und Wissensformen („Bildung“) - Kompetenzen (inkorporiertes kulturelles Kapital), die genau wie Schulabschlüsse und akademische Titel (institutionalisiertes kulturelles Kapital) im Unterschied zu ökonomischem Kapital personengebunden sind;

- soziales Kapital: Beziehungen zu anderen Akteuren (Netzwerke), die bei der Reproduktion von ökonomischem und kulturellem Kapital eingesetzt werden können;

- symbolisches Kapital: die Anerkennung der anderen drei Kapitalformen durch die Umwelt (Prestige, Renommee).

Kapital ist in jedem Fall „akkumulierte Arbeit“ (Bourdieu, 1983: 183). Der Arbeitsbegriff wird in den Wirtschaftswissenschaften anders verwendet als in der Soziologie, der Philosophie oder der Physik und ist schon deshalb problematisch, weil er "ein universeller Ausdruck für Lebenstätigkeit geworden ist" (Liessmann, 2000: 86). Wir leisten Beziehungs- und Erziehungsarbeit, Körper- und Erholungsarbeit, wir arbeiten an unserer Persönlichkeit und haben dadurch einen „Arbeitstag“, der „24 Stunden umfasst“ (Liessmann, 2000: 104). Bei Bourdieu ist der Arbeitsbegriff mit Aufwand und Mühe, mit Investitionen und mit (latentem) Zwang konnotiert. Natürlich kann man ökonomisches und kulturelles Kapital in objektiviertem Zustand erben, finden oder geschenkt bekom- 
men, Bourdieu hat aber immer wieder darauf hingewiesen, dass Kapital und damit die Verbesserung der eigenen Position im sozialen Raum „nur um den Preis von Arbeit, Anstrengungen und vor allem Zeit zu haben sind“ (Bourdieu, 1985: 13; vgl. Bourdieu, 1983: 186) - Zeit, in der man auf andere, weniger anstrengende Tätigkeiten verzichten muss. Auch den „Erwerb von Bildung“ bezahlt man „mit seiner Person“ - selbst in privilegierten Umgebungen, in denen „die Inkorporierung von kulturellem Kapital“ möglicherweise „völlig unbewusst“ und ungewollt abläuft (Bourdieu, 1983: 186f.). Auch wenn in jedem sozialen Feld eine andere Kapitalmischung den größten Handlungsspielraum verspricht, streben bei Bourdieu letztlich alle Menschen nach Kapital (vor allem nach ökonomischem und nach kulturellem Kapital), um sich von anderen zu unterscheiden und um ihre Position zu verbessern.

Wenn man diesem Ansatz folgt, dann ist danach zu fragen, wie die zehn Stunden, die der deutsche Durchschnittsbürger jeden Tag mit den Angeboten der Massenmedien verbringt (Reitze \& Ridder, 2006: 49f.), zum individuellen Kapitalfundus beitragen. Der Link zu den Unterhaltungstheorien, die in der Kommunikationswissenschaft diskutiert werden, liegt dabei nahe: Um meine Arbeitskraft optimal in ökonomisches Kapital umsetzen zu können, brauche ich Phasen der Erholung und Entspannung und das, was Früh als Abwechslung beschrieben hat (Früh, 2002; Früh \& Stiehler, 2003; Wünsch, 2006). Der hier verwendete Unterhaltungsbegriff ist allerdings nicht psychologisch fundiert und macht das Unterhaltungserleben nicht an "affektiven Prozessen“ (Vorderer, 1998: 690) oder an Informationsverarbeitungsprozessen fest (Früh, 2002), sondern am Bruch mit den Tätigkeiten, die nötig sind (und folglich nicht einfach umgangen werden können), um die eigene Existenz zu sichern. Zur Unterhaltung können all die Beschäftigungen werden, die uns von einer anderen Seite beanspruchen als die notwendigen Verrichtungen, die gleichzeitig für Vergnügen, Kurzweil und Zerstreuung sorgen und die folglich objektiv der Reproduktion der Arbeitskraft dienen, selbst wenn es subjektiv bei der als angenehm erlebten "Makroemotion“ Unterhaltung zunächst ,nur' um psychisches Wohlbefinden gehen sollte. Damit allein ist der extensive Umgang mit Medienangeboten ohnehin genauso wenig zu erklären wie mit dem Bedürfnis nach Überblickswissen (Brosius, 1995; Meyen, 2004: 125-129).

Übernimmt man die Terminologie Bourdieus, dann ist der Gegensatz von Unterhaltung weder Information noch Langeweile (Klaus, 1996), sondern Arbeit - Arbeit, die eingesetzt wird, um kulturelles Kapital zu erwerben und so die eigene Position im Spiel um Macht und Anerkennung zu festigen oder auszubauen. Bourdieu hat zwar behauptet, dass „ein Individuum die Zeit für die Akkumulation von kulturellem Kapital nur so lange ausdehnen“ könne, „wie ihm seine Familie freie, von ökonomischen Zwängen befreite Zeit" garantiere (Bourdieu, 1983: 188), diese verengte Sicht widerspricht aber den Grundannahmen des Habitus-Konzepts - ein Widerspruch, der auch damit zu erklären ist, dass der Begriff des „kulturellen Kapitals“ entwickelt wurde, um ungleiche Schulleistungen begreifen zu können (Bourdieu, 2001: 112). Bourdieus Gegenstände waren außerdem vor allem die kabylische Gesellschaft und das Frankreich der 1970er Jahre und nicht postmoderne Gesellschaften mit hoher Mobilität, sehr viel Freizeit und einer hohen Formalbildung, die keineswegs mehr ausschließlich vom ökonomischen Kapital abhängt. Natürlich gilt wie bei allen anderen Habitus-Bestandteilen auch für inkorporiertes kulturelles Kapital, dass frühe Spuren später nicht zu tilgen oder einfach zu überschreiben sind, der „Verinnerlichungsprozess“ (Bourdieu, 1983: 186) aber ist (zumindest hier und heute) keineswegs mit dem Ende der Ausbildungszeit abgeschlossen. Am Beispiel des „lohnabhängigen Kleinbürgertums" hat Bourdieu beschrieben, dass am inkorporierten kulturellen Kapital genau wie am Habitus insgesamt ständig gearbeitet 
wird. Der „gute Wille in Sachen Kultur“ drücke hier klar „das Streben nach Aufstieg“ aus (Bourdieu, 1987: 206). Obwohl es sich bei der Nutzung von Medienangeboten in der Regel nicht um bezahlte Erwerbsarbeit handelt, hat der hier verwendete Arbeitsbegriff schon deshalb ähnliche Merkmale und ähnliche (latente) Funktionen (Jahoda, 1982: 83f.; Beck, 2000: 51), weil am Ende die Akkumulation von (kulturellem) Kapital steht. Mediennutzung als Arbeit

- kostet Kraft und Zeit und ist mit dem Verzicht auf andere (weniger anstrengende und als angenehmer erlebte) Tätigkeiten verbunden,

- ist fremdbestimmt und unterliegt dem Zwang, sich an die alles ,beherrschende Realität' anzupassen,

- ist teilweise durch Spezialisierung gekennzeichnet,

- ist Quelle von Aktivität und stellt eine Zeitstruktur zur Verfügung,

- sorgt für Teilnahme am sozialen Leben außerhalb der Kernfamilie,

- gibt Ziel und Lebenssinn (Religionsersatz) und

- fördert soziale Anerkennung und persönliche Identität.

Viele der Befragten haben es als „Muss“ empfunden, über die Themen Bescheid zu wissen, die in der Gesellschaft oder im persönlichen Umfeld für wichtig gehalten und im Moment diskutiert werden. Die Tagesschan zum Beispiel wurde immer wieder als Pflichtprogramm beschrieben - vor allem von Personen, die man der Mittel- und der Oberschicht zurechnet und die sich in Feldern bewegen, in denen kulturelles Kapital besondere Profitchancen verspricht (vgl. Bourdieu, 1985: 10; 1998a: 40). Eine Bankkauffrau aus Baden-Württemberg, Jahrgang 1962, sagte, dass sie sich die Wirtschaftsnachrichten „antun“ müsse. Wenn der nächste Kunde komme und frage, wie es eigentlich Infineon gehe, dann könne sie nicht bloß die Überschrift gelesen haben. Auch ein PRBerater, Jahrgang 1973, meinte, er könne es sich „gar nicht leisten“, die Wirtschaftspresse nicht zu lesen. Um den Kunden Themen vorschlagen zu können, müsse man „auf der Höhe der Zeit“ sein, und auf Abendveranstaltungen mache es „einen sehr komischen Eindruck“, wenn man immerzu sagen müsse, dass man über dieses nicht Bescheid wisse und von jenem noch nie etwas gehört habe. Genau wie bei den anderen Bestandteilen des inkorporierten kulturellen Kapitals meint „auf der Höhe der Zeit sein“ hier nicht so sehr Wissenshäppchen, die man in ein Gespräch einbringen kann und in der Regel schnell wieder vergisst, sondern die generelle kulturelle Handlungskompetenz (vgl. Bourdieu, 2001: 115). Eine Abteilungsleiterin, Jahrgang 1959, sprach über ihr großes Interesse an Politik, über Joschka Fischer und Kofi Annan und sagte, dass man diese Dinge genauso wissen müsse wie das, was in ihrem Heimat-Bundesland passiert. Sie liest dazu regelmäßig den Spiegel, das Time Magazin, die Frankfurter Allgemeine und die Financial Times Deutschland, hat das Frühstücksfernsehen laufen, hört im Auto ein öffentlich-rechtliches Wortprogramm und hält das Internet vor allem bei Auslandsreisen für unverzichtbar. Diese Konzentration auf Medienwissen wird verständlicher, wenn man weiß, dass diese Frau nicht studiert hat und ihr damit eine wichtige Form kulturellen Kapitals fehlt. Im Interview hat sie selbst darauf hingewiesen, dass die Leute „verwundert gucken“ würden, wenn sie sage, dass sie keinen Hochschulabschluss habe. Ersatz ist zum Beispiel das Feuilleton der FAZ. Diesen Teil habe sie jahrelang weggeworfen, „weil das sehr anspruchsvoll ist und auch sehr gewöhnungsbedürftig“. Der erste Schritt sei dann der Vorsatz gewesen, jeden Tag mindestens einen Artikel zu lesen. Das habe ihr „den Zugang zum Kulturellen“ eröffnet. Inzwischen finde sie dort immerzu interessante Sachen und studiere das komplette „Buch“.

Medienwissen und Medienmenü werden von dieser Abteilungsleiterin auch als Di- 
stinktionsmerkmale eingesetzt: Sie liest gern und mit Leichtigkeit Texte, die längst nicht jeder versteht. Bourdieu hat jeden Konsumakt als „Unterscheidungszeichen“ beschrieben, völlig unabhängig davon, ob dahinter die entsprechende Absicht steht oder nicht (Bourdieu, 1985: 21; Bourdieu, 1987: 120, 355), und auch unabhängig davon, ob sich die jeweilige „Praxis“ in der Öffentlichkeit vollzieht oder in der Privatsphäre. Genau wie Automarke, Wohnform oder Kleidung signalisieren die Medienangebote, die ein Mensch nutzt und über die er bei Bedarf folglich sprechen kann, welche soziale Position er anstrebt und welcher Gruppe er sich verbunden fühlt, welche Einstellungen und Werte er teilt und welche nicht. Die Investition in diese Form von kulturellem Kapital ist vor allem deshalb nötig, weil der Wohlstandsschub in den westlichen Gesellschaften und die Öffnung der Bildungswege in den vergangenen drei bis vier Jahrzehnten zentrale Unterschiede nivelliert haben - vor allem beim Besitz von ökonomischem Kapital, beim Zugang zu akademischer Bildung und beim Status durch Herkunft. Wer über andere (seltenere) Formen kulturellen Kapitals verfügt (wie zum Beispiel Künstler), dürfte weniger an Medienwissen interessiert sein. Ein Musiker, Jahrgang 1949, lehnte zum Beispiel zu viel Zeitunglesen ab, weil man dadurch „verbildet“ werde. Das kulturelle Kapital dieses Musikers hat einen „Seltenheitswert“, aus dem er „Extraprofite“ ziehen und so auf extensive Mediennutzung verzichten kann.

Der Norm Informiertsein können sich vor allem diejenigen nicht entziehen, die sich nicht in solchen speziellen Feldern bewegen wie der Musiker und trotzdem eine gehobene soziale Position bekleiden oder aufsteigen wollen. Wer diesen Wunsch nicht hat und in der gesellschaftlichen Hierarchie (im „sozialen Raum“) unten oder am Rand steht, kann eher darauf verzichten, die Tageszeitung „durchzuackern“ und sich nach dem Tatort bei Sabine Christiansen oder Anne Will ein Update für den Montagmorgen zu holen. Mediennutzung als Arbeit ist folglich ein Preis, der für sozialen Aufstieg zu zahlen ist, und Mediennutzung als Unterhaltung umgekehrt eine Entschädigung für untergeordnete Positionen. Dies erklärt, warum eine Ministeriumsangestellte, Jahrgang 1959, die für einen Staatssekretär die Reden zu schreiben hat und deshalb von sich verlangt, ein „eigenes Bild“ von der deutschen Geschichte zu haben, vor allem über die Stoff-Fülle geklagt hat, die das Fernsehen zu diesem Thema bietet („Um Gottes Willen. Das sollst Du jetzt eigentlich auch noch alles wissen“). Diese Frau wünschte sich vor allem kurze Sendungen („gut sortiert", mit einem "Zeitlimit“) und "große Themen“ - Themen, mit denen möglichst viele andere Kollegen, Freunde und Zufallsbegegnungen etwas anfangen können und an denen deshalb die eigene Kompetenz gemessen werden kann. Indikator für die Bedeutung eines Themas ist dabei auch seine mediale Präsenz. Was auf allen Kanälen läuft, darf man schon deshalb nicht verpassen, weil wir das Bedürfnis nach Anschlusskommunikation haben - ein Bedürfnis, das sich am besten mit Themen befriedigen lässt, bei denen man sicher sein kann, dass die anderen davon auch etwas mitbekommen haben. Eine Dolmetscherin, Jahrgang 1945, die bei der Kirch-Gruppe in der Filmproduktion gearbeitet hat und kurz vor dem Interview arbeitslos geworden war, sagte, dass sie am liebsten Bücher lese, die gerade einen Preis gewonnen hätten. Da einer Handlung wie Mediennutzung in der Regel ein ganzes Bündel von Motiven zugrunde liegen dürfte und jede Sendung und jede Zeitung (auch gleichzeitig) ganz unterschiedliche Bedürfnisse befriedigen kann, sind Unterhaltung und Arbeit nicht als Gegensätze zu verstehen, die sich ausschließen, sondern eher als Anfangs- und Endpunkte einer Skala, auf denen der Umgang mit den Medienangeboten jeweils verortet werden kann.

Bourdieus Theorie erklärt auch, warum Mediennutzung stets mit Identitätsmanagement verbunden sein dürfte, mit dem Versuch, den eigenen Wert zu taxieren, die eigene Lage zu bestimmen und das Meinungsklima zu erkunden (Festinger, 1954; Scherer 
\& Wirth, 2002; Hartmann, Schramm \& Klimmt, 2004; Noelle-Neumann, 1980) - egal ob die Zuwendung zu Medienangeboten eher als Arbeit zu verstehen ist oder eher als Unterhaltung. Bourdieu geht davon aus, dass wir die „soziale Struktur in ihrer Gesamtheit" über unseren „Sinn für die eigene Stellung im sozialen Raum“ beherrschen. Jeder Mensch habe ein „Gespür dafür, was man ,sich erlauben' darf und was nicht“ (Bourdieu, 1985: 17f.). Bourdieu verwendet Goffmans „sense of one's place“ zwar, um zu erklären, warum die Akteure die Welt so hinnehmen, wie sie ist („gesellschaftlich konstituierter Selbsterhaltungstrieb“; Bourdieu, 1985: 18), das Interviewmaterial liefert aber zugleich zahlreiche Belege für die These, dass der „Sinn für Grenzen“ und damit für die eigene Identität vor allem über die Beobachtung von Medienfiguren geschärft wird. Für Belege sei an dieser Stelle auf die Typologie verwiesen, die im dritten Abschnitt präsentiert wird.

In bestimmten sozialen Feldern kann das Wissen über andere Personen, über Handlungsmöglichkeiten und Lebenswege auch zu kulturellem Kapital werden (zum Beispiel dort, wo man als Ratgeber gefragt sein möchte). Ein Polizist aus Essen, Jahrgang 1982, der die Fachzeitschrift Psychologie heute abonniert hat und die Aufsätze nach einem ausgeklügelten System archiviert, erzählte, wie dankbar ein älterer Kollege die Kopien aus dem Blatt aufgenommen hat, als bei einem seiner Kinder ein Aufmerksamkeitsdefizitsyndrom diagnostiziert wurde. Der junge Polizist liest neben Stern und Spiegel regelmäßig die Satirezeitschriften Eulenspiegel und Titanic und kauft sich außerdem gesellschaftskritische Bücher, weil die meisten Kollegen diese Art von Literatur nicht kennen und er auf der Dienststelle schon oft Anerkennung für entsprechende Empfehlungen geerntet hat („symbolisches Kapital“).

Dem sozialen Aufstieg oder der Absicherung der Position durch die Akkumulation von kulturellem Kapital in Form von Medienwissen sind allerdings Grenzen gesetzt. Diese Grenzen hängen zwar auch davon ab, in welchem sozialen Feld man sich bewegt, in der (gebrochenen) Hierarchie der Kapitalformen aber steht das Geld ganz oben. Schon seit der Marienthal-Studie ist bekannt, dass Arbeitslose zum Beispiel keineswegs anfangen, sich weiterzubilden. Im Forschungsbericht der Gruppe um Lazarsfeld war vom „Einschrumpfen aller Lebensäußerungen“ die Rede. Die Einwohner gingen seltener in die Bibliothek als früher und lasen weniger Zeitung, sie verzichteten auf das Radio, auf Fahrten in das nahe Wien und auf das Gasthaus und interessierten sich kaum noch für die Politik (Jahoda et al., 1975). Über wie viel kulturelles Kapital ein Arbeitsloser auch verfügen kann: In einer Gesellschaft, in der bezahlte Erwerbsarbeit über das Ansehen und über den persönlichen Spielraum entscheidet, nützt ihm dies ohnehin nichts. Das Interviewmaterial lässt vermuten, dass dieser Zusammenhang für andere ökonomisch benachteiligte Gruppen ganz ähnlich gilt (Rentner, Mütter mit Kleinkindern, Alleinerziehende, Ausländer).

\section{Mediennutzer in Deutschland: Eine Typologie}

Eine Typologie soll Ordnung in eine unüberschaubare Vielfalt bringen und schlaglichtartig Unterschiede zwischen den einzelnen Elementen erhellen. Ein Typus steht dabei für eine Gruppe von Menschen, die bestimmte Merkmale gemeinsam haben. Der Haupteinwand gegen jede Typenbildung heißt Willkür. Die Ordnung, die herauskommt, wird von den Vorgaben des Forschers bestimmt, ohne dass er sicher sein kann, alle und vor allem die richtigen Merkmale erfasst zu haben. Diese ,Gestaltungsmöglichkeit' ist ein Grund, warum es gerade im Bereich Mediennutzung eine ganze Reihe von konkurrierenden Typologien gibt. Haas und Brosius haben von einer „Mode“ gesprochen und sogar von 
„Ikonen der modernen Mediaforschung“. „Typen“ würden „voll im Trend“ liegen und vor allem für die Hoffnung stehen, „menschliches Verhalten und Erleben“ besser erklären zu können als soziodemografische Variablen wie Alter, Bildung, Einkommen oder Geschlecht. Allerdings seien Typologien bisher den Beweis schuldig geblieben, mehr zu können als die herkömmlichen Erklärungs- und Prognose-Instrumente. Mit viel Aufwand werde etwas produziert, was vielleicht als "Marketing- oder Verkaufsargument“ tauge, aber kein „sinnvolles Tool der Mediaplanung“ sei (Haas \& Brosius, 2006: 159, 176-178; vgl. Haas, 2007: 117-121).

Die Typologie, die hier präsentiert wird, verfolgt ein theoretisches Ziel. Sie fragt danach, wovon die Kommunikationsbedürfnisse und die Bedeutung abhängen, die Medienangebote im Leben des Einzelnen haben, und erhebt so den Anspruch, menschliches Verhalten erklären und vorhersagen zu können. Wie bei jeder anderen Typologie handelt es sich dabei um eine Konstruktion. Die Einteilung stützt sich auf die ersten beiden offenen Kategorien, die die Auswertung geleitet haben (Muster der Mediennutzung, Bewertung des Medienangebots). Diese Kategorien wurden mit Hilfe der Befunde, die in Abschnitt 2 präsentiert wurden, auf zwei operationalisierbare Kriterien verdichtet:

- Arbeitsorientierung bei der Mediennutzung. Dieser Begriff zielt nicht auf den Job, den ein Befragter gerade hat und für den er vielleicht auch Zeitung lesen muss, sondern auf den Erwerb kulturellen Kapitals und damit auf einen langfristig angelegten Prozess. Wie in Abschnitt 2 diskutiert wurde, lässt sich der Umgang mit Medienangeboten jeweils auf einer Skala zwischen den Endpunkten Arbeit und Unterhaltung verorten. Indiz für eine starke Arbeitsorientierung war neben den entsprechenden Äußerungen im Interviewmaterial („,durchackern“, „studieren“) die Nutzung von Angeboten, die als ,anstrengend' gelten und gerade deshalb ein hohes Prestige haben (überregionale Tageszeitungen, Auslandspresse, politische Magazine, dezidierte Informationsangebote im Fernsehen, Wortprogramme im Radio, klassische Musik). Für eine starke Unterhaltungsorientierung stand umgekehrt ein Medienmenü, in dem solche Zutaten fehlen und das vor allem aus kommerziellen Funkprogrammen besteht. Natürlich lässt sich aus den Merkmalen des Angebots nicht auf die Nutzungsmotive schließen (zumal jedes Angebot eine ganze Reihe von Bedürfnissen befriedigen und aus ganz verschiedenen Gründen genutzt werden kann), mit Bourdieu (1987: 355) wurde aber davon ausgegangen, dass in jedem „Kulturgut“ die „Distinktionsbeziehung objektiv angelegt“ ist. Die Menschen müssen das nicht bei jedem einzelnen Nutzungsakt wissen oder wollen, sie haben aber eine Vorstellung, welche Angebote „wertvoll“ sind und welche nicht (Staab \& Hocker, 1994: 162f.).

- Bedentung von Medienangeboten im Alltag. Genau wie bei der Arbeitsorientierung handelt es sich auch bei diesem Kriterium um eine Interpretationsleistung, die sich zum einen auf den Umfang der Mediennutzung stützt (Zeitdauer als Indiz für Bedeutung) und zum anderen auf die Bindung an die Angebote der Massenmedien, die für den einen, unverzichtbar' sein können (weil er sie als Distinktionsmerkmale oder für das Identitätsmanagement benötigt) und für den anderen, vollkommen unwichtig‘. Die Auswertung hat gezeigt, dass von dieser Bindung zugleich das Urteil über die Qualität der Medien abhängt.

Bei allem Wissen darum, dass Typenbildung immer zur Vereinfachung zwingt und dass die Grenzen zwischen Typen stets fließend sind, lassen sich mit Hilfe dieser beiden Kriterien sechs Formen des Umgangs mit den Angeboten der Massenmedien unterscheiden. Die Position dieser sechs Typen in der Abbildung ist dabei nicht mit dem Lineal nachzumessen, sondern lediglich als ,Sehhilfe' gedacht. Über Größenordnungen (wie 
viele Deutsche gehören zu den einzelnen Typen) kann die Abbildung keine Auskunft geben, da sich die Typologie auf ein qualitatives Verfahren stützt, das zwar den Anspruch hat, Verallgemeinerungen zuzulassen und alle Varianten zu entdecken, aber trotzdem nicht repräsentativ ist. An den Rekrutierungshürden für Tiefeninterviews scheitern vor allem Menschen, die formal niedrig gebildet sind und den unteren Schichten angehören. Deshalb nützt es überhaupt nichts zu wissen, dass etwa dem Typ ,Die Profis' 31 Befragte zugeordnet wurden und dem Typ ,Die Genügsamen' nur 16. In der Grundgesamtheit (erwachsene Deutsche, die nicht mehr zur Schule gehen) ist die Verteilung möglicherweise genau andersherum.

\section{Abbildung 2: Mediennutzer-Typen in Deutschland}

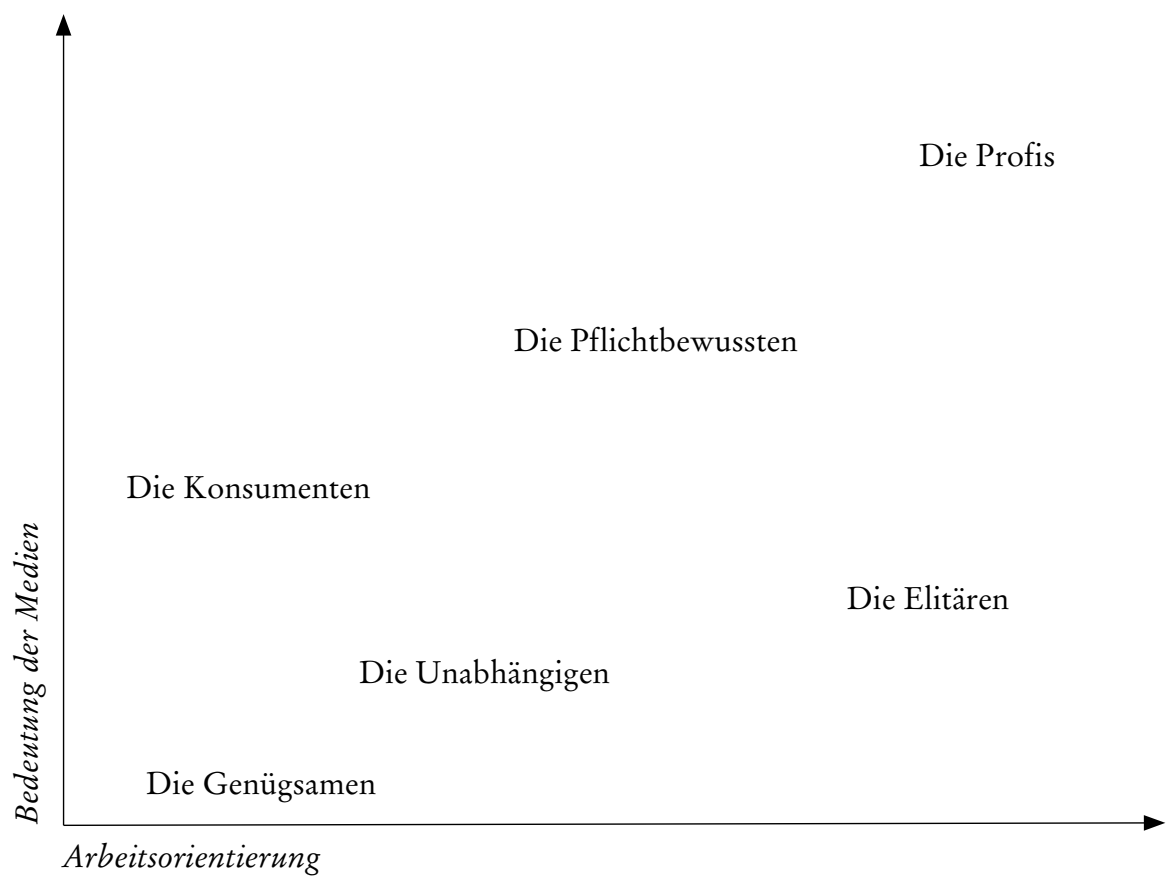

\section{Typ 1: Die Genügsamen}

Die Genügsamen sind zufrieden mit dem, was ihnen die Massenmedien bieten. Genauer gesagt: Sie machen sich über dieses Angebot eigentlich keine Gedanken. Ihr Medienmenü wird durch die Alltagsstrukturen bestimmt oder durch den Partner. Nur einer der Befragten, die diesem Typ zugeordnet wurden, nutzt das Internet, und keiner hat gesagt, dass er ins Kino geht. Das Radio wird als Geräuschkulisse genutzt („so alte Lieder; was halt so kommt", Arbeiter, Jahrgang 1929) und das Fernsehen als Abendgast und als Schlafmittel („,schneller geht es nicht“, der gleiche Arbeiter). Die meisten Genügsamen haben zwar eine Tageszeitung abonniert, es bleibt aber beim ,Blättern', beim schnellen Blick auf die ersten zwei, drei Seiten und vielleicht noch beim Lokalteil. Eine Hotelbetreiberin, Jahrgang 1964, die für ihr Haus drei Zeitungen hält und auch gleich morgens hineinschaut („wenn die Gäste frühstücken“), hat auf die Frage nach dem „Lesen“ mit 
dem Kopf geschüttelt. „Wirklich nur selektiv. Das ist ein kleines Hotel. Da ist es nicht so wichtig, dass man schon in der Früh die Weltlage umrissen hat". Obwohl die Genügsamen die Politikberichterstattung nur wahrnehmen, um sicher zu sein, dass nichts Bedrohliches passiert ist (wofür manchen auch Nachrichtensendungen reichen), fühlen sie sich ,gut informiert'. Sie sei niemand, der "den ganzen Abend“ $n$-tv sehen müsse, sagte eine Hausfrau, Jahrgang 1968. „Das ist mir auch zu viel“.

Die Genügsamen sind in den unteren Bereichen der gesellschaftlichen Hierarchie zu finden und haben in der Regel einen kleinen Verkehrskreis - beschränkt auf die Wohnung oder das Haus, auf die Kernfamilie, auf einige wenige Personen im unmittelbaren Umfeld, vielleicht auf den Weg zur Arbeitsstelle. Sie haben entweder mit dem Leben weitgehend abgeschlossen (was nicht nur auf sehr alte Menschen zutreffen kann, sondern auch auf psychisch und körperlich Kranke) oder sind durch ihren Alltag so ausgelastet, dass selbst für die Nutzung von Unterhaltungsangeboten nur wenig Zeit und Kraft bleibt. Früher galt dies vor allem in den Sommermonaten für die Landbevölkerung, heute zum Beispiel für junge Mütter und Selbstständige in bestimmten Bereichen (Autohäuser, Gaststätten, Läden). Wichtig scheint in jedem Fall, dass die Genügsamen nicht mehr sozial aufsteigen wollen - entweder, weil die körperlichen und intellektuellen Voraussetzungen dies ohnehin nicht zulassen, oder weil die maximale Position bereits erreicht oder übertroffen wurde. Da der Aufstiegswunsch fehlt, sind gesellschaftliche Erwartungen wie die Norm, informiert zu sein, für diesen Typ weitgehend unbedeutend. Es lohnt sich deshalb für ihn nicht, kulturelles Kapital in Form von Medienwissen zu erwerben.

\section{Typ 2: Die Konsumenten}

Das Medienmenü der Konsumenten beschränkt sich in aller Regel auf werbefinanzierte Funkkanäle (bei manchen Männern kommen PC-Spiele, Play Station oder Premiere dazu), und das Interesse an Politik ist minimal. „Es betrifft mich nicht wirklich“, sagte eine Frisöse, Jahrgang 1965, die zwei Kinder im Alter von sieben und 14 Jahren hat. "Schon in der Schule habe ich das nicht gemocht. Ich habe es gehasst. All das mit Politik und so". Wer bei den TV-Duellen vor den letzten beiden Bundestagswahlen „dabei gewesen“ ist, weiß diese Frau nicht. „Ich kann da einfach nicht zuhören. Ich kann dazu nichts sagen“. Gewählt hat sie trotzdem: „um nicht doof da zu stehen“. Wie die meisten Konsumenten hat die Frisöse kaum Print-Abonnements („nur TV Spielfilm“) und keine Lust zum Zeitungslesen („eigentlich gar nicht“). Einige der jüngeren Befragten, die zu diesem Typ gehören, sehen eine Tageszeitung, wenn sie ihre Eltern besuchen, und manche Konsumenten haben Blätter im Büro. Genutzt wird diese Chance aber nur, „wenn Sachen auf der Welt passieren, die wichtig für mich sind“ (Betreiberin eines Kosmetikstudios, Jahrgang 1970). Die Kosmetikerin kauft an solchen Tagen einfach ein Boulevardblatt. Eine chemisch-technische Assistentin, Jahrgang 1983, der es ähnlich geht, findet selbst in der Abendzeitung oder in der $t z$ viele Artikel, mit denen sie überhaupt nichts anfangen kann. Kommentare? „Sollte ich lesen, aber ich kann mich da nie aufraffen“. Eine sächsische Ingenieurin, Jahrgang 1967, hat die Samstagsausgabe ihrer Lokalzeitung abonniert. „In der Woche lohnt sich das einfach nicht. Da stapelt sich das nur".

Die meisten Konsumenten sind in den 1970er und 1980er Jahren aufgewachsen, in einer Zeit, in der es kommerzielles Fernsehen bereits gab oder in der die Debatte um solche Angebote auch das öffentlich-rechtliche schon zu einem Unterhaltungsfernsehen gemacht hatte. Wie die Genügsamen sind die Konsumenten eher in den unteren Schich- 
ten der Gesellschaft zu finden und haben wenig ökonomisches und oft auch wenig kulturelles Kapital: Frauen und (etwas weniger) Männer, die ihr Geld mit den Händen verdienen (etwa als Kellnerinnen, Verkäuferinnen, Pfleger oder Kraftfahrer), Studenten, Selbständige und Freiberufler, die sich mit Mühe über Wasser halten. Während die Genügsamen durch Alter, Krankheit oder kleine Kinder an einige wenige Orte gebunden sind, ist der Aktionsradius der Konsumenten schon deshalb größer, weil sie kämpfen müssen, um zu überleben, und weil sie teilweise noch auf Partnersuche sind. Dieser Typ interessiert sich zwar noch weniger für Politik als die Genügsamen, Medienangebote sind für ihn aber wichtiger - natürlich zur Entspannung, aber auch als Beleg, dazuzugehören und wenigstens im Unterhaltungsbereich Bescheid zu wissen, oder als Hilfsmittel bei der Anbahnung von Kontakten. Ihren Verzicht auf Informationen aus Politik und Wirtschaft und auf so genannte Qualitätsangebote verteidigen die meisten Konsumenten mit heftiger Medienschelte und mit der Angst vor starken Medienwirkungen. Einige der Befragten sahen ihre Kinder in Gefahr und einige die Alten („die halten das teilweise wirklich für Realität“; Bürokauffrau, Jahrgang 1967). Manche breiteten im Interview auch Verschwörungstheorien aus. „Ich bin fast zu 100 Prozent überzeugt, dass mehr als die Hälfte verschwiegen wird“, sagte eine Angestellte, ebenfalls Jahrgang 1967. „Absichtlich, aus welchen Gründen auch immer. Um das Volk nicht zu beunruhigen“.

\section{Typ 3: Die Unabbängigen}

Für diesen Typ haben Medienangebote kaum eine größere Bedeutung als für die Genügsamen, und eigentlich lesen die Unabhängigen nicht mehr Zeitung als die Konsumenten. Manche haben gar kein Abonnement und manche nur am Wochenende, manche lesen die Zeitung nur online, und ein Lehrer, Jahrgang 1953, der nebenbei Privatdozent an einer Universität ist, wartet so lange, bis sein Zeitungsstapel eine Zugfahrt füllt. Die Unabhängigen: Das sind Menschen, die vergleichsweise viel ökonomisches Kapital haben (Firmeninhaber, Anwälte, Apotheker, Ärzte, Gastronomen, Führungskräfte) oder über kulturelles Kapital verfügen, das sie von der Mehrheit der Bevölkerung abhebt (Künstler, Wissenschaftler, Pfarrer). Die Aufzählung der Berufe deutet bereits darauf hin, dass dieser Typ in der ,Mitte des Lebens' steht (Alter: 35 bis 60 Jahre), seinen Platz in diesem Leben gefunden hat (vor allem in den oberen Schichten der Gesellschaft) - dort, wo es nicht mehr nötig ist, sich mit den Distinktionsmerkmalen Medienwissen und Medienmenü den eigenen Status zu beweisen, oder dort, wo es genügt, dass das unmittelbare Umfeld meine Position akzeptiert (etwa bei Künstlern). Zum kulturellen Kapital der Unabhängigen gehört, dass sie die Vorgänge in der Medienlandschaft verfolgen, die einzelnen Angebote einordnen und Qualität folglich schätzen können. Trotz solchen Wissens spielen Medienangebote im Leben der Unabhängigen eine untergeordnete Rolle - nicht vergleichbar jedenfalls mit der Aufgabe, die man lösen muss, oder mit der Kunst, der man sich verschrieben hat. Ein Gastwirt, Jahrgang 1966, der „sieben Tage die Woche“ in seinem Betrieb ist, sagte, er habe sich „für das Lokal hier entschieden“. Damit sei er eigentlich „mehr oder weniger von der Außenwelt abgeschnitten“ und brauche sich weder mit „lokalen Sachen“ zu beschäftigen noch mit der „deutschen Politik“.

Medienangebote sind für die Unabhängigen auch deshalb nicht so wichtig, weil sie nicht auf dieses Unterhaltungsmittel angewiesen sind - entweder weil sie sich Alternativen leisten können oder weil ihr ,Job' kein Job ist, der nach Abwechslung ruft, sondern Erfüllung. Wenn die Unabhängigen Medienangebote nutzen, dann in erster Linie für die Arbeit. Der Musiker, Jahrgang 1949, der Angst hat, beim Zeitungslesen „verbildet“ zu werden, setzt sich zum Beispiel „konzentriert“ vor sein Radiogerät, um in öffentlich- 
rechtlichen Spartenkanälen und in einem Ausbildungsprogramm nach „Hörmaterial“ zu suchen - fast so, als ob er „in einem Konzertsaal wäre“. Wer in so herausgehobene Positionen aufsteigt wie die meisten der Befragten, die zu diesem Typ gehören, kann sich natürlich den Erwartungen der Mitmenschen nicht ganz entziehen. Vor allem bei den Unternehmern und den Führungskräften gehört es zum Habitus, auf dem Laufenden zu sein. Die Unabhängigen versuchen aber, dieser Norm mit möglichst geringem Aufwand gerecht zu werden. Eine Wissenschaftlerin, Jahrgang 1960, die keine Zeitung liest und im Fernsehen bestenfalls $C N N$ einschaltet, sagte, sie brauche im Radio „eigentlich keine Musik“, sondern „Informationen“. Wenn sie BS aktuell anmache, bekomme sie das „immer" - schnell und ohne auf eine „bestimmte Zeit" angewiesen zu sein. Ein Drehbuchschreiber, Jahrgang 1972, gab offen zu, sich in der Politik „nicht so auszukennen“. Er komme damit aber „ganz gut zurecht“.

\section{Typ 4: Die Pflichtberwussten}

Die Pflichtbewussten sind längst nicht so locker wie der Drehbuchschreiber. Sich nicht auszukennen und das auch noch zuzugeben, ist für diesen Typ schwer vorstellbar. Die Pflichtbewussten haben eine Tageszeitung abonniert und lesen sie in der Regel jeden Tag ausführlich. Diese Zeitung muss kein Qualitätsblatt sein. Ein Polizist, Jahrgang 1956, der in einer Kleinstadt bei München lebt und auch die Süddeutsche als Heimatzeitung kaufen könnte, sagte, dass man ja „ein BWL-Studium“ brauche, um dort zum Beispiel „den Wirtschaftsteil zu kapieren“. Ihm persönlich sei das alles auch zu „langatmig“. „Mir kommt es auf kurze und prägnante Informationen an. Ich will einfach schnell durchkommen. Aber bitte nicht auf dem Niveau der Bild-Zeitung". Die Pflichtbewussten erleben die Norm, informiert zu sein, als Muss, und haben den Anspruch, dieser Norm zu genügen und sich so nach unten abzugrenzen - egal ob sie sich selbst zum Beispiel für Politik interessieren oder nicht. Extreme Vertreterin ist eine Fernsehjournalistin, Jahrgang 1962, die Zeitungen eigentlich seit ihrer Kindheit hasst, aber sich „notgedrungen“" damit plagt. Wenn die Pflichtbewussten den eigenen Ansprüchen nicht genügen, dann haben sie ein schlechtes Gewissen.

Das Wissen um die Erwartungen der Umwelt hat dazu geführt, dass die meisten Befragten, die zu diesem Typ gehören, in den Interviews kommerzielle Fernsehprogramme zunächst rundweg abgelehnt haben. Das heißt aber nicht, dass sie ausschließlich öffentlich-rechtliche Kanäle einschalten - gerade bei den jüngeren Leuten oft eher im Gegenteil. Abgesehen vom Schimpfen auf die „Verdummungswelle“, die sich aus dem Fernsehapparat über die „Normalbevölkerung“ ergießt (Ingenieur im Ruhestand, Jahrgang 1938), hielt sich die Medienkritik in den Gesprächen mit den Pflichtbewussten in Grenzen. Dieser Typ fühlt sich gut informiert - manchmal sogar zu gut. „Es gibt einfach zu viel“, klagte eine Abteilungsleiterin, Jahrgang 1963, die einen siebenjährigen Sohn hat. „Wenn man vor so einem Kiosk steht und dort Hunderte von Titeln sieht, dann fragt man sich, oh Gott, was soll ich da jetzt nehmen“. Weniger wäre für diese Frau tatsächlich mehr - mehr Sicherheit, auch wirklich alles Wichtige erfasst zu haben. Die Pflichtbewussten sind im Durchschnitt etwas älter als die Konsumenten und stehen in der ,Mitte der Gesellschaft'. Sie haben eine Position erreicht, die ihren Fähigkeiten entspricht oder diese leicht übersteigt, und wollen diese Position absichern - auch mit Hilfe von Medienwissen: Polizisten und Techniker, Lehrer, Ministerialbeamte und Bankangestellte, Abteilungsleiter und Versicherungskaufleute. Dazu kommen Senioren, die aus dem Statuskampf schon deshalb noch nicht ausgeschieden sind, weil sie noch fit 
sind, und die vor dem Ausscheiden aus dem Arbeitsleben eine Position hatten, die es sich zu verteidigen lohnt.

\section{Typ 5: Die Profis}

Dieser Typ geht virtuos mit den Angeboten der Massenmedien um und nutzt ein unerhört breites Menü, das von überregionalen Tageszeitungen und Politikmagazinen über Informationssendungen im Fernsehen und Nischenprogrammen im Radio bis zu Büchern und Kino reicht und zu dem selbstverständlich das Internet gehört. Solche ,Profis ${ }^{\varsigma}$ sind zum einen Menschen, die in den Medien oder für die Medien arbeiten (vor allem Journalisten in Leitungspositionen, in aktuellen Redaktionen oder mit Karriereambitionen und PR-Fachleute), sowie Menschen, bei denen Medienwissen auch deshalb zum Berufsprofil gehört, weil ihr Erfolg stark davon abhängt, wie sie selbst oder ihr Arbeitgeber in der öffentlichen Kommunikation dargestellt werden. Dazu kommt eine zweite Gruppe von Profis: Menschen, die sehr gut ausgebildet sind oder über hervorragende intellektuelle Voraussetzungen verfügen, die aber ausgebremst wurden und (noch) nicht die Position erreicht haben, die möglich gewesen wäre. Diese Profis nutzen Medienangebote, um ihren Statusanspruch zu signalisieren, um ein Gebiet zu haben, auf dem sie ihren Vorgesetzten und beruflich erfolgreicheren Personen überlegen sind, und einfach auch, um die Zeit mit ,Sinn' zu füllen, die ihnen ein Job lässt, der sie nicht voll fordert.

Der relative Misserfolg im Kampf um ökonomisches Kapital kann dabei ganz unterschiedliche Ursachen haben (die Geburt der Kinder und ein Partner, der genug Geld hat; eine Mutter, die gepflegt werden muss; die Pleite des Betriebs, in dem man sich hochdienen wollte; politischer Idealismus in der Jugend, der sich nicht mit den Machtverhältnissen vertragen hat; ein Job, der schon in jungen Jahren lebenslange Sicherheit garantiert und so die Aufstiegsmotivation dämpft, und natürlich die Unwägbarkeiten des Lebens - vor allem bei schwer planbaren Karrieren wie etwa in der Wissenschaft oder in der Kunst). Für diese Gruppe von Profis ist Medienwissen oft nicht der einzige Aufstiegsersatz. Viele engagieren sich in der Gesellschaft - in der Lokalpolitik, in der Kleinkultur, in der Nachbarschaft, in speziellen Interessengruppen. Eine Krankenpflegerin, Jahrgang 1944, die gerade arbeitslos geworden war und auf ihre Rente wartete, leitet einen Kreis, der sich der Aufklärung über Borreliose verschrieben hat. Dazu gehört, die Berichterstattung auf Fehler zu überprüfen, Berichtigungen und Pressetexte $\mathrm{zu}$ schicken. Politik hat diese Frau „schon von der Jugend an unheimlich interessiert“. Nach der Ausbildung war sie 20 Jahre daheim, um die Kinder großzuziehen, und hat danach noch einmal neu in einer Reha-Klinik angefangen. In der Zeitung beginnt sie heute nicht mit dem Lokalteil („das macht mein Mann, aber ich nicht“), sondern mit der Politik, und im Wirtschaftsteil liest sie „immer jede Zeile“. Dazu kommen Phoenix („aktuelle Debatten aus dem Bundestag und so“), Report, Monitor und Panorama sowie „ab und zu“ Spiegel oder Focus, weil der Politikteil der Regionalzeitungen „ein bisschen schwach" sei. „Ich denke einfach, es ist wichtig, dass man Bescheid weiß. Sonst kann man nirgends mitreden. Ich hasse diese Proleten, die am Tisch sitzen, nur daherreden und eigentlich gar keine Ahnung haben". Der Umgang mit Medienangeboten wird für manche Profis zur Sucht. Der Stellenwert dieser Beschäftigung ist auf jeden Fall so groß, dass die entsprechenden Nutzungsmuster auch den Wechsel vom Berufsleben in das Rentenalter überdauern können. 


\section{Typ 6: Die Elitären}

Die Elitären sind nicht sehr weit weg von den Unabhängigen und in gewisser Weise sogar eine Juniorausgabe. Für beide Typen haben Medienangebote eine eher geringe Bedeutung. Beide meiden vor allem Unterhaltungsangebote in Hörfunk und Fernsehen (nur in „schwachen Momenten“; Bibliothekarin, Jahrgang 1974), und beide gehen mit der Berichterstattung sehr kritisch um. Ein Student, Jahrgang 1980, der in der SPD aktiv ist und „seit sechs, sieben Jahren keinen Fernseher mehr benutzt" hat, begründete diese „negative Grundhaltung“ damit, dass er sich „nicht steuern“ lassen wolle. „Allein die Idee ist mir zu blöd. Ich müsste dann ja da sein, um das zu sehen“. Während die Unabhängigen aber so gut wie keine Zeitung lesen und versuchen, sich mit möglichst geringem Aufwand eine Art ,Grundinformation' zu sichern, sind Medienangebote für die Elitären zumindest als Distinktionsmerkmal wichtig - vor allem zur Abgrenzung nach unten. Die Befragten, die diesem Typ zugeordnet wurden, lesen die taz, das Handelsblatt oder ausländische Tageszeitungen, sie nutzen im Radio Zuhörprogramme und haben Zeitschriften wie Emma, Psychologie heute, Ökotest oder Brand eins abonniert. Worum es dabei geht, hat eine Politologin, Jahrgang 1980, auf den Punkt gebracht. Es mache überhaupt keinen Sinn, „Proleten“ oder „,irgendwelchen arschigen Kindern aus dem Osten“ eine Zeitung wie die taz unter die Nase zu halten und ihnen „zu erklären, dass Ausländer auch Menschen“ seien. „Wahrscheinlich wissen die überhaupt nicht, was die taz ist".

Die Elitären sind noch auf dem Weg. Sie stehen in der gesellschaftlichen Hierarchie zwar relativ weit unten, sind aber entweder gerade in einen attraktiven Beruf eingestiegen oder dabei, andere Formen kulturellen Kapitals zu erwerben (etwa eine akademische Ausbildung). Die Elitären haben deshalb die Hoffnung, in absehbarer Zeit so viel ökonomisches Kapital erwerben zu können, dass ihre Statuswünsche erfüllt werden. Eine solche Aufstiegschance sehen keineswegs nur Studierende oder Absolventen. Eine Ägyptologin, Jahrgang 1958, die als Sekretärin arbeitet, um Ausgrabungen und wissenschaftliches Schreiben zu finanzieren, ist noch nicht zu alt, um auf eine Professur berufen zu werden oder wenigstens wissenschaftlichen Ruhm zu ernten. Da die Elitären (noch) nicht mit Haut und Haaren in die Mühlen des Berufsalltags geraten sind und ihre Zeit schon wegen des Aufstiegswunsches anders investieren, scheinen sie wenig Unterhaltung und Abwechslung zu benötigen. Vielleicht ist es Zufall, dass diesem Typ kein einziger Rentner zugeordnet wurde. Auch wenn der Volksmund sagt, dass zuletzt die Hoffnung stirbt - der Aufstiegswunsch ist früher tot.

\section{Fazit}

Der theoretische Hintergrund, vor dem die Untersuchung gelaufen ist, leitet natürlich den Blick auf die Faktoren, die Mediennutzung beeinflussen. Rosengrens Lebensstilmodell und Bourdieus Kapital-Theorie schreiben der Position, die der Einzelne in der gesellschaftlichen Hierarchie einnimmt, jeweils eine große Bedeutung zu. Da die beiden Typologisierungsmerkmale dieser Grundannahme folgen, ist gar kein Ergebnis denkbar, in dem der Status der Befragten keine Rolle spielt. Das Kriterium ,Arbeitsorientierung" steht für die Akkumulation kulturellen Kapitals und das Kriterium ,Bedeutung' für die Nutzung von Medienangeboten als Distinktionsmerkmale und für das Identitätsmanagement. Die hier präsentierte Typologie ist auch als empirischer Beleg zu lesen, dass positionelle und individuelle Merkmale und Bedingungen tatsächlich den Umgang mit Medienangeboten beeinflussen und dass Medienmenüs und Medienwissen als Mittel 
gesehen werden, die eigene Position zu halten oder zu verbessern. Dies gilt auch für einen Typ wie die Konsumenten, die zwar wenig prestigeträchtige Funkkanäle nutzen, sich dadurch aber zumindest von den Genügsamen abheben und gleichzeitig die Zugehörigkeit zu bestimmten Gruppen sichern sowie (über Medienschelte) die eigene Lage genauso wie den Verzicht auf andere Angebote legitimieren können.

Zugleich beantwortet diese Typologie nicht nur die Frage nach dem Zusammenhang zwischen Status und Mediennutzung, sondern gibt auch Hinweise darauf, welche Faktoren den Umgang mit Medienangeboten tatsächlich beeinflussen. So wichtig die soziale Position ist: Mindestens genauso wichtig ist die Bewertung, die die Befragten mit dieser Position verbinden. Die Typologie der Mediennutzer legt nahe, dass der Umgang mit Medienangeboten vor allem davon geprägt wird, wie man seine bisherige Karriere und die eigenen Zukunftsaussichten einschätzt. Dieses Urteil wiederum wird von zahlreichen positionellen und individuellen Merkmalen beeinflusst:

- Geschlecht. Es ist zu vermuten, dass Frauen zumindest tendenziell etwas eher zufrieden sind, weil die Erwartungen an ihre Karriere nach wie vor geringer sein dürften.

- Lebensphase. Viele der Befragten, die im Moment zu den ,Elitären' gehören, werden ihre Mediennutzungsmuster in den nächsten Jahrzehnten ändern und dann entweder zu den ,Unabhängigen' gehören, zu den ,Pflichtbewussten' oder zu den ,Profis'. Von der Lebensphase hängt zum Teil auch der Umgang mit kommerziellen Funkprogrammen und natürlich mit dem Internet ab (Stichwort: Mediensozialisation; vgl. Peiser, 1996).

- Handlungsspielraum im Beruf. Die „Unabhängigen“ differenzieren die These, dass Mediennutzung als Arbeit ein Preis sei, der für sozialen Aufstieg zu zahlen ist. In Berufen mit hohem Sozialprestige, bei großem Einkommen oder Vermögen und im Feld Kunst sind die Distinktionsmerkmale Medienwissen und Medienmenü weitgehend überflüssig.

- Herkunft. Der Anspruch an sich selbst wird durch die Sozialisation geprägt, durch die Erwartungen des Elternhauses, durch die Region, aus der man kommt. Viele Ostdeutsche sehen sich zum Beispiel als Bürger ,zweiter Klasse - mit Folgen für ihre Mediennutzung.

- Persönliches Umfeld. Bourdieus Feldtheorie geht davon aus, dass in jedem Feld eine andere Kapitalmischung den größten Handlungsspielraum verspricht (man vergleiche nur das Medienwissen, das Künstler benötigen, mit dem von Journalisten).

- Alltagsbelastung. Wer Kinder, Kranke oder Eltern zu betreuen hat, wer durch ein Haus oder durch ein Ehrenamt gefordert wird, der hat nicht nur weniger Zeit für die Nutzung von Medienangeboten als jemand, der sich nur auf seinen Job konzentrieren kann. Er wird seine Position auch anders beurteilen.

- Mobilität. Der Nationalökonom Karl Knies hat schon vor 150 Jahren die „Vorbedingung" für jeden „Nachrichtenverkehr" beschrieben: Menschen, die nicht persönlich miteinander sprechen können, aber „Interesse aneinander haben“ (Knies, 1857: 54, 56). Die ,Genügsamen' sind nicht weit herumgekommen in ihrem Leben und haben die Menschen, die für sie wichtig sind, in ihrer Nähe.

- Persönliche (auch intellektuelle) Voraussetzungen: Spätestens in der Schule entwickelt jeder eine Vorstellung von der Position, die für ihn erreichbar ist. Eine „Konsumentin“, Jahrgang 1983, sagte zwar, dass sie vielleicht ins „Lehramt“ wechseln wolle, BS aktuell ist ihr aber zu anstrengend und der Duden das wichtigste Mittel auf dem Weg zur „Allgemeinbildung“. Warum sollte diese Frau mit sich hadern, wenn sie doch chemisch-technische Assistentin bleibt?

- Beruflicher Bezug zu den Medien. Die funktionale Differenzierung der Gesellschaft 
und die dadurch wachsende Bedeutung öffentlicher Kommunikation haben in gewisser Weise einen Selbstverstärkereffekt. Immer mehr Menschen arbeiten im Journalismus, in der Öffentlichkeitsarbeit und in der Werbung, und genau diese Menschen sind es, die schon von Berufs wegen gezwungen sind, sich ständig mit Medienangeboten zu befassen.

Wenn durch Mediennutzung kulturelles Kapital akkumuliert wird und wenn Medienwissen und Medienmenüs zu Distinktionsmerkmalen geworden sind, die genau wie Automarke, Wohnform oder Kleidung signalisieren, welche soziale Position ich anstrebe und welchen Gruppen ich mich verbunden fühle, welche Einstellungen und Werte ich teile und welche nicht, dann darf Mediennutzung nicht nur im Kontext von funktionalen Alternativen und mit Blick auf den Alltag betrachtet werden. Wer Mediennutzung erklären und vorhersagen will, sollte zugleich den Kapitalbesitz der einzelnen Akteure einbeziehen sowie die sozialen Felder, in denen sie sich bewegen.

\section{Literatur}

Averbeck, Stefanie (2003): Pierre Bourdieu und die Journalismusforschung in Frankreich. Vorbemerkungen zum Aufsatz von Gilles Bastin. In: Publizistik, 48. Jg., S. 253-257.

Bastin, Gilles (2003): Ein Objekt, das sich verweigert: Der Journalismus in der Soziologie Pierre Bourdieus. Einige Bemerkungen über das ,journalistische Feld“. In: Publizistik, 48. Jg., S. 258273.

Bausinger, Hermann (1984): Media, Technology and Daily Life. In: Media, Culture and Society, Vol. 6, S. 343-351.

Beck, Ulrich (2000): Wohin führt der Weg, der mit dem Ende der Vollbeschäftigungsgesellschaft beginnt? In: Beck, Ulrich (Hrsg.): Die Zukunft von Arbeit und Demokratie. Frankfurt: Suhrkamp, S. 7-66.

Blumler, Jay G.; Katz, Elihu (1974): The uses of mass communications: current perspectives on gratification research. Beverly Hills: Sage.

Bourdieu, Pierre (1976): Entwurf einer Theorie der Praxis auf der ethnologischen Grundlage der kabylischen Gesellschaft. Frankfurt: Suhrkamp.

Bourdieu, Pierre (1983): Ökonomisches Kapital, kulturelles Kapital, soziales Kapital. In: Kreckel, Reinhard (Hrsg.): Soziale Ungleichheiten. Göttingen: Schwartz \& Co., S. 183-198.

Bourdieu, Pierre (1985): Sozialer Raum und „Klassen“. Zwei Vorlesungen. Frankfurt: Suhrkamp.

Bourdieu, Pierre (1987): Die feinen Unterschiede. Kritik der gesellschaftlichen Urteilskraft. Frankfurt: Suhrkamp.

Bourdieu, Pierre (1998a): Praktische Vernunft. Zur Theorie des Handelns. Frankfurt: Suhrkamp.

Bourdieu, Pierre (1998b): Über das Fernsehen. Frankfurt: Suhrkamp.

Bourdieu, Pierre (2001): Die drei Formen kulturellen Kapitals. In: Bourdieu, Pierre: Wie die Kultur zum Bauern kommt. Über Bildung, Schule und Politik. Hamburg: VSA, S. 112-120.

Bourdieu, Pierre (2002): Ein soziologischer Selbstversuch. Frankfurt: Suhrkamp.

Brosius, Hans-Bernd (1995): Alltagsrationalität in der Nachrichtenrezeption. Ein Modell zur Wahrnehmung und Verarbeitung von Nachrichteninhalten. Opladen: Westdeutscher Verlag.

Bryant, Jennings; Vorderer, Peter (2006) (Eds.): Psychology of entertainment. Mahwah, NJ: Lawrence Erlbaum Associates.

Charlton, Michael; Neumann-Braun, Klaus (1988): Mediensozialisation im Kontext. Der Beitrag des Kontextualismus und der Strukturanalyse für die Medienforschung. In: Publizistik, 33. Jg., S. 297-315.

Cornelißen, Waltraud (1998): Fernsehgebrauch und Geschlecht. Zur Rolle des Fernsehens im Alltag von Frauen und Männern. Opladen: Westdeutscher Verlag.

Festinger, Leon (1954): A Theory of Social Comparison Processes. In: Human Relations, Vol. 7, S. $117-140$. 
Früh, Werner (2002): Unterhaltung durch das Fernsehen. Eine molare Theorie. Konstanz: UVK. Früh, Werner; Stiehler, Hans-Jörg (2003) (Hrsg.): Theorie der Unterhaltung. Ein interdisziplinärer Diskurs. Köln: Halem.

Fuchs-Heinritz, Werner (2000): Biographische Forschung. Eine Einführung in Praxis und Methoden. Opladen: Westdeutscher Verlag.

Haas, Alexander (2007): Medienmenüs. Der Zusammenhang zwischen Mediennutzung, SINUSMilieus und Soziodemographie. München: R. Fischer.

Haas, Alexander; Brosius, Hans-Bernd (2006): Typen gibt's! Zur Brauchbarkeit von Typologien in der Mediaforschung. In: Focus-Jahrbuch, S. 159-179.

Hartmann, Tilo; Schramm, Holger; Klimmt, Christoph (2004): Personenorientierte Medienrezeption: Ein Zwei-Ebenen-Modell parasozialer Interaktionen. In: Publizistik, 49. Jg., S. 25-47.

Hepp, Andreas (1998): Fernsehaneignung und Alltagsgespräche. Fernsehnutzung aus der Perspektive der Cultural Studies. Opladen: Westdeutscher Verlag.

Huber, Nathalie; Meyen, Michael (2006): Medien im Alltag. Qualitative Studien zu Nutzungsmotiven und zur Bedeutung von Medienangeboten. Münster: Lit.

Jahoda, Marie (1982): Employment and Unemployment: A Social-Psychological Analysis. Cambridge: Cambridge University Press.

Jahoda, Marie; Lazarsfeld, Paul F.; Zeisel, Hans (1975): Die Arbeitslosen von Marienthal. Frankfurt: Suhrkamp.

Jensen, Klaus Bruhn; Rosengren, Karl Erik (1990): Five traditions in Search of the audience. In: European Journal of Communication, Vol. 5, S. 207-239.

Johansson, Thomas; Miegel, Frederik (1992): Do the Right Thing. Lifestyle and Identity in Contemporary Youth Culture. Stockholm: Almquist \& Wiksell.

Klaus, Elisabeth (1996): Der Gegensatz von Information ist Desinformation, der Gegensatz von Unterhaltung ist Langeweile. In: Rundfunk und Fernsehen, 44. Jg., S. 402-417.

Knies, Karl (1857): Der Telegraph als Verkehrsmittel. Mit Erörterungen über den Nachrichtenverkehr überhaupt. Tübingen: Laupp.

Krotz, Friedrich (2005): Neue Theorien entwickeln. Eine Einführung in die Grounded Theory, die heuristische Sozialforschung und die Ethnographie anhand von Beispielen aus der Kommunikationsforschung. Köln: Halem.

Lamnek, Siegfried (1998): Gruppendiskussion. Theorie und Praxis. Weinheim: Psychologie Verlags Union.

Liessmann, Konrad Paul (2000): Im Schweiße Deines Angesichts. In: Beck, Ulrich (Hrsg.): Die Zukunft von Arbeit und Demokratie. Frankfurt: Suhrkamp, S. 85-107.

Mayring, Philipp (2002): Einführung in die qualitative Sozialforschung. Eine Anleitung zu qualitativem Denken. München: Psychologie Verlags Union.

Meyen, Michael (2004): Mediennutzung. Mediaforschung, Medienfunktionen, Nutzungsmuster. Konstanz: UVK.

Mikos, Lothar (1994): Fernsehen im Erleben der Zuschauer. Vom lustvollen Umgang mit einem populären Medium. München: Quintessenz.

Noelle-Neumann, Elisabeth (1980): Die Schweigespirale. Öffentliche Meinung - unsere soziale Haut. München, Zürich: R. Piper.

Noelle-Neumann, Elisabeth; Petersen, Thomas (1998): Alle, nicht jeder. Einführung in die Methoden der Demoskopie. München: Deutscher Taschenbuch Verlag.

Peiser, Wolfram (1996): Die Fernsehgeneration. Eine empirische Untersuchung ihrer Mediennutzung und Medienbewertung. Wiesbaden: Westdeutscher Verlag.

Reitze, Helmut; Ridder, Christa-Maria (2006): Massenkommunikation VII. Eine Langzeitstudie zur Mediennutzung und Medienbewertung 1964-2005. Baden-Baden: Nomos.

Rosengren, Karl Erik (1994): Culture, Media and Society: Agency and Structure, Stability and Change. In: Rosengren, Karl Erik (Ed.): Media Effects and Beyond. London: Routledge, S. 328.

Rosengren, Karl Erik (1995): Substantive Theories and Formal Models - Bourdieu Confronted. In: European Journal of Communication, Vol. 10, S. 7-39. 
Rössler, Patrick; Kubisch, Susanne; Gehrau, Volker (2002) (Hrsg.): Empirische Perspektiven der Rezeptionsforschung. München: R. Fischer.

Rubin, Alan (2002): The uses-and-gratifications perspective of media effects. In: Bryant, Jennings; Zillmann, Dolf (Eds.): Media Effects: Advances in Theory and Research. Hillsdale: Lawrence Erlbaum, S. 525-548.

Scherer, Helmut; Wirth, Werner (2002): Ich chatte - wer bin ich? Identität und Selbstdarstellung in virtuellen Kommunikationssituationen. In: Medien \& Kommunikationswissenschaft, 50. Jg., S. 337-358.

Schmidt, Christiane (2005): Analyse von Leitfadeninterviews. In: Flick, Uwe; von Kardorff, Ernst; Steinke, Ines (Hrsg.): Qualitative Forschung. Ein Handbuch. Reinbek: Rowohlt, S. 447-456.

Schulze, Gerhard (2000): Die Erlebnisgesellschaft. Kultursoziologie der Gegenwart. Frankfurt, New York: Campus.

Schweiger, Wolfgang (2005): Gibt es einen transmedialen Nutzungsstil? Theoretische Überlegungen und empirische Hinweise. In: Publizistik, 50. Jg., S. 173-200.

Staab, Joachim Friedrich; Hocker, Ursula (1994): Fernsehen im Blick der Zuschauer. Ergebnisse einer qualitativen Pilotstudie zur Analyse von Rezeptionsmustern. In: Publizistik, 39. Jg., S. $160-174$.

Vorderer, Peter (1998): Unterhaltung durch Fernsehen: Welche Rolle spielen parasoziale Beziehungen zwischen Zuschauern und Fernsehakteuren? In: Klingler, Walter; Roters, Gunnar; Zöllner, Oliver (Hrsg.): Fernsehforschung in Deutschland. Themen - Akteure - Methoden. Baden-Baden: Nomos, S. 689-707.

Weiß, Ralph (2001): Fern-Sehen im Alltag. Zur Sozialpsychologie der Medienrezeption. Wiesbaden: Westdeutscher Verlag.

Wirth, Werner; Schramm, Holger; Gehrau, Volker (2006) (Hrsg.): Unterhaltung durch Medien. Theorie und Messung. Köln: Halem.

Wünsch, Carsten (2006): Unterhaltungserleben. Ein hierarchisches Zwei-Ebenen-Modell affektivkognitiver Informationsverarbeitung. Köln: Halem. 